\title{
The Mach-Zehnder interferometer and photon dualism
}

Paul Klevgard

Paul A. Klevgard, "The Mach-Zehnder interferometer and photon dualism," Proc. SPIE 11481, Light in Nature VIII, 114810B (21 August 2020); doi: $10.1117 / 12.2575213$

SPIE. Event: SPIE Optical Engineering + Applications, 2020, Online Only 


\title{
The Mach-Zehnder interferometer and photon dualism
}

\author{
Paul A. Klevgard \\ Sandia National Laboratory, Ret. \\ pklevgard@gmail.com
}

\begin{abstract}
The Mach-Zehnder Interferometer (MZI) is used to illustrate the long-standing wave-particle duality problem. How can the photon divide at the first beam splitter and yet terminate on either arm with its undiminished energy? Why is which-way (welcher weg) information incompatible with wave interference? How do we explain Wheeler's delayed choice experiment?

The position adopted is that the photon has two identities, one supporting particle features and the other wave features. There is photon kinetic energy that never splits (on half-silvered mirrors) or diffracts (in pinholes or slits). Then there is photon probability waves that do diffract and can reinforce or cancel. Interpretations of these two identities are suggested.
\end{abstract}

Keywords: $\quad$ Mach-Zehnder Interferometer; photon; wave-particle dualism; duality; delayed choice; which-way; Wheeler; double slit

\section{CONTENTS}

1. THE MZI AND PHOTONIC EXPERIMENTATION

2. THE PHOTON REQUIRES DUAL IDENTITIES FOR DUAL ATTTRIBUTES

2.1 Rest mass invariant over time; photon KE invariant over space

3. FIRST PHOTON IDENTITY: PHOTON KINETIC ENERGY

4. SECOND PHOTON IDENTITY: MASS STORED VIA E $=\mathrm{MC}^{2}$

4.1 Objective probability waves

4.2 Collapse of probability waves

5. PHOTON AS PARTICLE?

6. HOW THE PHOTON TRAVERSES THE MZI

7. CONCLUSION

8. CONCEPTUAL OBSTACLES

9. APPENDIX A

Light in Nature VIII, edited by Vasudevan Lakshminarayanan, Katherine Creath, Joseph A. Shaw, Proc. of SPIE Vol. 11481, 114810B · () 2020 SPIE

CCC code: $0277-786 X / 20 / \$ 21 \cdot$ doi: $10.1117 / 12.2575213$ 


\section{THE MZI AND PHOTONIC EXPERIMENTATION}

The Mach-Zehnder interferometer (MZI) has proven to be a boon for physicists eager to perform a publishable experiment, especially in the last 20 or 30 years. It is inexpensive, table-top physics and modern photonics has provided for a multitude of variations and techniques.

The modern MZI experimenter has an array of possible sources and devices that can be employed. They include: lasers, fiber optics, amplifiers, photodiodes, quantum dots, vibrating mirrors, nested beam splitters, random generators, entangled photons, etc. Some MZI experiments get very complicated. An example by A. Danan, et al. [1] features multiple (nested) vibrating mirrors and beam splitters. The idea is to frequency "tag" the photons on certain paths "to see where they have been." The conclusions reached - in support of the two-state vector formulism (TSVF) have proved controversial and the back and forth dispute cannot be covered here. ${ }^{1}$ Certainly the best known use of the MZI is the Wheeler delayed choice experiment, something that will be discussed presently.

Unfortunately, MZI experiments have not given us a clear resolution of the wave versus particle duality. Theoretical explanations for duality have also been unsatisfactory. The Copenhagen Interpretation leaves the MZI as a mystery, insisting that quantal behavior in general cannot be modeled or explained using classical concepts. Some theorists apply the wave function to the photon but this raises a couple of issues. First, the wave function is mathematical in configuration space and not physical. And second, you cannot write a Schrödinger equation for something lacking rest mass.

All of this seems a bit discouraging. Nevertheless, some progress can be made if we focus on the nature of photon kinetic energy (KE).

\section{THE PHOTON REQUIRES DUAL IDENTITIES FOR DUAL ATTTRIBUTES}

Our usage of a single word, photon, to refer to quantized radiation leads us to believe there is a single object that has, like any (material) object familiar to us, specific attributes. Unfortunately in this case the attributes are contradictory: discrete (particle-like) vs. continuous (wave-like). The way out of this impasse is to retain the contradictory attributes but give up on the concept of the photon as an object with but a single identity. For the photon we need to look for a dualism of identities to match up with the dualism of attributes. Toward that end we look at what is unique about objects or entities that progress in only one dimension: the photon progressing in space and the inertial particle progressing (persisting) in time.

When a photon enters the MZI we know the photon probability of reception gets divided by the first beam splitter such that each arm of the MZI has a 50\% chance of photon reception. But if received on one arm or the other, the photon terminates with its undivided kinetic energy (KE). No space device - pinhole, slit, half-silvered mirror - can fractionate the KE of a photon. Why should this be true? Does it tell us that we don't understand radiation KE very well?

\subsection{Rest mass invariant over time; photon KE invariant over space}

Symmetry is an important topic in physics. Gauge (measurement) symmetry has an entity remaining invariant when the measurement reference basis changes. A simple example is the Galilean principle of relativity where the laws of physics are invariant for uniform motion. Even simpler is the invariance of an entity stationary in one dimension

\footnotetext{
${ }^{1}$ For the controversy see notes 4-33 of [2].
} 
while advancing (progressing) in the opposite dimension. This can be an existing entity remaining invariant as time progresses, or an occurring entity (i.e., a photon) remaining invariant when its space location changes. Invariance (symmetry) transcends dimensions (space, time), categories (existence, occurrence) and entity basis (mass, energy).

It is revealing when we look at invariance for observations made on particles and on photons. Both are measurable entities and both are quantized. They are mirror images of each other in several ways. The photon is stationary in time since anything at the velocity of light suffers infinite time dilation. The force free particle is stationary in space within its own inertial system. Being stationary in space makes the particle all rest mass with no KE. Conversely, the time-stationary photon is all KE with no rest mass. The space stationary particle and the time stationary photon are "pure" entities in that they do not mix KE with rest mass. Symmetry suggests they will be invariant for observers stationed along the dimension where they progress, space for the photon, time for the particle.

Assume there is a rest mass particle stationary in space. As time passes, successive observers see or measure the same particle. The particle (entity) and its mass remains invariant over successive observations.

Observation invariance over time for material objects is something we take for granted; it is the law of identity viewed temporally. Observers over time share the same, unchanging material object; successive observations have the material object in common. We simply say that time and its passage are orthogonal to existing, space-residing objects. And something is orthogonal to a dimension if it does not reside in that dimension.

\section{- Inertial material objects/particles reside (occupy an interval) in space and are common to all observations as the object progresses in time.}

Imagine now a single photon moving past a number of space-separated observers. Each observer is a possible recipient of this photon as it passes and the same is true for the next observer. Each observer in space would measure the same photon with its characteristic frequency and polarization. The photon (entity) and its KE remains invariant over successive (possible) observations.

Now suppose this single photon passes through a pinhole or double slit. The photon diffracts and now has multiple paths to reach a target screen. If we position observers on each of these paths they can no longer be assured of photon reception; each path only affords an observer a defined (objective) probability of reception. But one observer on one path will receive the photon and find it to be the same (the only) photon with the same photon KE as originally generated. Photon entity invariance over space paths is preserved for photon KE but not for photon probability. This difference is telling us something.

\section{FIRST PHOTON IDENTITY: PHOTON KE}

The invariance of entities (particles, photons) when progressing through a dimension (time or space) reflects a common situation: a stationary entity residing in one dimension while that entity progresses in the alternate dimension. The entity is present in the alternate dimension but in a progressing way; this means it does not occupy an interval in the alternate dimension while it does so in the residing dimension. Your desk progresses (persists) in time so it does not occupy an interval there; but it does occupy an interval (volume) in space where it resides. This is how space and time are orthogonal for stationary entities. Stationary entities can only (reside in) occupy an interval/volume in one dimension; for moving entities see Appendix A.

We identify entities by their kinetic (unstored) identity despite the fact that they store something (as a potential identity). We identify a particle as "mass" and write equations for it even though it stores energy. This is why we can identify a photon as "photon KE" even though it stores relativistic mass; equations for the photon $(\mathrm{E}=h f)$ are written for its energy (for its kinetic identity). 
Since the orthogonal nature of space and time accounts for particle invariance during time progression, we may assume it does the same for photon KE invariance during space progression. This requires the invariant item to reside in (occupy) but one dimension so that it is unaffected by progression in the alternate, orthogonal dimension. This means that oscillatory photon KE must reside in the time dimension making it common to (shared by) observers on all available space locations (paths).

- Inertial rest mass does not rarefy along time paths because it resides in space.

- Photon KE does not rarefy along space paths because it resides in time.

- Photon KE in time cannot be fractionated by material, space-residing devices: pinholes, slits or halfsilvered mirrors.

- By residing in time, photon $\mathrm{KE}$ is orthogonal to photon space paths making this energy common to (shared by) all possible space observers.

Entities can only reside in a dimension by occupying an interval there. A quantized particle entails (rest) mass which requires (occupies) a space volume. A quantized photon entails oscillatory-cyclical KE which requires (occupies) a time interval. Photon KE cannot be a mere quantity; it must involve oscillation cycles occupying time. The concept of photon $\mathrm{KE}$ as a mere quantity without oscillation and no presence in a dimension is wrong.

Photon energy is created when work is done upon a charge. Photon energy can also be generated by the release of stored work (an electron changing atomic orbits). Doing or releasing work to produce radiation creates photon KE residing in time as pure oscillation; this is not the oscillation of something material or existing. If you ask how pure oscillation can exist, you are betraying your bias for a material reality that only exists (and in space). Photon energy oscillation occurs and it does so in time. Occurrence in and of itself is the ontological counterpart of existence in and of itself; the former is time-residing energy, the latter is space-residing mass. We need to grant the realm of energy/occurrence equal standing with the realm of mass/existence just as $\mathrm{E}=\mathrm{mc}^{2}$ implies.

- Photon KE is matter-free oscillation residing in time.

- It constitutes the photon's first identity.

Rest mass is an entity occupying space. Letting photon KE be an entity occupying time presents two conceptual challenges: 1) broadening our current concept of what is real; and 2) envisioning photon KE as pure oscillation.

Reality: We live in a world of material objects that occupy space. Our concept of reality is rooted in existence, mass and space. But radiation is based on occurrence, energy and time; trying to explain it based on our existing material world only leads to paradoxes.

Entities require a presence in a dimension. We regard the photon as an entity but then try to place its KE in space as a quantity (a payload). Regarding photon KE as a mere quantity traveling in space is an adaptation of a $19^{\text {th }}$ century concept characterizing matter-in-motion. Relativity and quantum mechanics made equality foundational: space with time and mass with energy $\left(\mathrm{E}=\mathrm{mc}^{2}\right)$. This means that quantized radiation $\mathrm{KE}$ is an entity just as quantized mass is an entity; they are mirror existence/occurrence images of each other. Quantized energy entities in time are the ontological counterparts of quantized mass entities in space. 
Pure oscillation: $\quad$ Physicists have embraced the oscillation of nothing or the oscillation from nothing: vacuum state fluctuation is an essential part of QFT. But this latter oscillation resides in space (naturally), appears randomly and creates (virtual, transient) particles that cannot be measured directly. This is unlike the oscillation of photon KE which can be measured, doesn't depend upon particles, hypothetical or real, and whose origin is real work.

The notion of an immaterial, oscillatory energy occurrence residing in time is no more problematic than an unmeasurable, transient, harmonic/oscillatory, virtual particle residing in space. And it completes particle/photon entity symmetry.

\subsection{Photon KE mimics particle impact}

Quantized mass resides in space and it is reasonable to argue that quantized energy (photon KE) resides in time. More difficult is the concept of pure occurrence (i.e., photon KE) being common for multiple space observers; this is a central concept in what follows. It identifies a new way that something may be present in space.

\section{- Pure occurrence in time is orthogonal to, and available for, multiple space-located observers}

- The inverse is: my (pure existence) desk is available to, and common for, multiple time observers

The photon's first identity operates (oscillates) in time. It is the photon's second identity (next section) that actively progresses in space. Because photon $\mathrm{KE}$ is common to space paths it is available for probabilistic release on those paths. But being in time also places some limitations on how photon KE can interact with matter.

Since the KE of a photon resides in time while the rest mass of a target resides in space, they are orthogonal to each other. With one occupying time and the other occupying space, the only way they can intersect is at a joint timeand-space point, namely an event since the latter requires both rest mass and KE. Hence time-residing photon KE can release to (intersect) an orthogonal dimension (space) only discretely, that is, at a space point thereby mimicking particle impact. This energy transfer is the "discrete" aspect of photon behavior; it is also random on an individual basis. All of this is a direct consequence of photon KE residing in time. Of course everyone wants to interpret photon $\mathrm{KE}$ reception as particle impact; but it is part of wisdom to question obvious 'solutions' that lead to paradoxes.

- Because photon KE resides in (occupies) time while rest mass resides in (occupies) space, the only way they can intersect is via a reception event that is discrete in both dimensions.

- Such an event is invariably interpreted as particle impact to conform to our concept of reality as limited to existence, mass and space.

\section{SECOND PHOTON IDENTITY: MASS STORED VIA E $=\mathrm{MC}^{2}$}

Our first photon identity, kinetic energy (KE), accounts for a number of photon attributes. These include: 1) oscillation; 2) non- rarefying energy available on diverging space paths; and 3) quantization, i.e., occurrence (a cycle) is whole just as existence (a particle) is whole.

This leaves a number of attributes for our second identity to contribute: 1) probability of photon reception; 2) spreading and progressing on all available space paths; and 3) collapse of what fills those space paths. 
Our look at symmetry and invariance showed that photon KE doesn't rarefy on diverging space paths while probability of reception does. Photon reception involves the transferring of kinetic energy from radiation to matter; from the realm of occurrence/time to the realm of existence/space (to a material target). The probabilistic nature of this transfer points to something latent ready to emerge. Something that facilitates the energy transfer but whose space presence depends upon photon KE itself. Such an intimate dependency must be that of $\mathrm{E}=\mathrm{mc}^{2}$ storage. We conclude that the photon's second identity is its potential (stored, relativistic) mass. ${ }^{2}$

\section{- The photon has two identities: kinetic in time and potential in space.}

A photon's first identity is oscillatory energy which is kinetic (unstored), operates (oscillates and resides) in time and is common to space paths. Its alternate, stored, orthogonal identity is the inverse of all this: potential, operates (progresses) in space and being particular (not common) to space paths. Because photon KE oscillates so does its potential (relativistic) mass. Since the latter progresses in space while oscillating, it has the (continuous) waveform.

We have seen (preceding section) that the photon's kinetic identity yields the photon's particle-like nature, namely termination at a point. It is the photon's potential identity that yields the "continuous" aspect of photon behavior permitting wave interference. ${ }^{3}$

The two photon identities function in different dimensions but both of them occur: photon KE as oscillation; photon potential (relativistic) mass as space-advancing waveform. Each has a "reflected" presence in the alternate dimension. Photon KE oscillation in time is impressed upon the photon's potential identity in space. In turn, the potential identity in space determines the transfer possibilities of a photon's KE in time. Neither identity in one dimension is without some shared presence or influence in the alternate dimension. This reflects the fact that the two identities constitute a single $\mathrm{E}=\mathrm{mc}^{2}$ quantum (host and that which is stored).

- Photon KE is pure occurrence entity in time; particle rest mass is pure existence entity in space; each is a kinetic identity; each has a potential (stored) identity.

- Photon KE occurs and resides in time making it common to those space paths its potential identity traverses.

- Photon potential (relativistic) mass also occurs and progresses and rarefies as a waveform on multiple space paths. Its local intensity determines probability of photon $\mathrm{KE}$ reception.

Physicists deny photon potential (relativistic) mass a space presence; to them it is merely a quantity explaining photon momentum. Photon potential (relativistic) mass has suffered the same fate at the hands of physicists as photon $\mathrm{KE}$; both are regarded as mere quantities without a presence in a dimension. This view is wrong; it dates from the $19^{\text {th }}$ century.

\footnotetext{
${ }^{2}$ Potential (relativistic) mass is out of favor these days with many physicists, largely for pedagogical reasons (“don't confuse students!'). Some wish to replace relativistic mass with energy arguing that the latter sustains relativistic mass and therefore relativistic mass is the same as (kinetic) energy. This argument is not convincing. Stored thermal energy sustains a mass increment in the body that hosts it and we don't say that thermal energy is the same as mass. In these pages stored mass or potential mass will be used as the equivalent of relativistic mass. But they all refer to the same thing: the mass a photon has. If something can be measured we should be loathe to deprecate it.[3]

${ }^{3}$ Commentators place the photon's wave nature and particle nature on equal footing. They fail to notice that particle-like behavior depends on KE transfer but wave behavior depends on the potential for reception (probability). The two are kinetic vs. potential and related by $\mathrm{E}=\mathrm{mc}^{2}$.
} 
The (kinetic) energy and (potential, relativistic) mass measures of the photon have always been interpreted as dependent, quantitative attributes of a single object with the object itself (the "photon") residing in space and time. This accords with our conception of reality as populated with existing objects located in both space and time like ourselves and particles and fields. But a photon doesn't conform to this concept (this ontology). First, it can't have a defined location in space; it is said to "travel" all paths but actually being in time it is common to all paths. And second, it is stationary in time so it does not progress in time as we humans and our desks and chairs do.

In reality, photon $K E$-as-entity oscillates in time while its potential (relativistic) mass counterpart progresses along all available paths in space. Trying to ascribe this division of labor to one undifferentiated object (a "photon") is doomed to failure. Equally futile, at least ontologically, is applying our material world, existing constructs (particle, field) to the realm of occurring radiation.

Photon potential (relativistic) mass progresses in space at the speed of light while sharing in the oscillation of its opposite (energy) number; this space-presence plus velocity and oscillation create the probability wave character of the photon. ${ }^{4}$ This waveform's space presence is real but in an occurring potential, hence probabilistic way. It is continuous in space and can disperse and rarefy there; but via interference, wave crests can superpose and reinforce. Our physical instruments cannot capture or measure this wave directly; we only receive photon KE or momentum. Nonetheless, from experiments we can infer two of the unusual properties of photon potential (relativistic) mass. It is: 1) a wave of "objective probability [4];" and 2) capable of instantaneous collapse.

\subsection{Objective probability waves}

The diffraction pattern of coherent photons passing through a pinhole (the representation is a so-called Airy pattern) can be predicted from a relatively few parameters. The mathematics yields areas of high and low wave intensity on a target screen. No one doubts that the mathematics is modeling something real. The mistake is to regard it as modeling the photon as a unitary object; rather it is only modeling the space-progressing identity of the photon, namely probabilistic photon potential (relativistic) mass. A photon has a set amount of potential (relativistic) mass to cover available paths in space. As potential mass waves interfere, path distribution changes and regions of high and low intensity are created, The release rate on any photon space path is proportional to the local intensity of the waveform's potential (relativistic) mass.

The potential (relativistic) mass of photon KE is stored (latent) and has the waveform making it continuous in space. When you model something stored and continuous in one dimension (space in this case), the measure you get is a continuous release rate for discrete events appearing in the alternate dimension. Hence the release rate is probabilistic and the events themselves (photon KE reception) are individually random. This is a direct consequence of source and destination: 1) being ontologically opposite entities; and 2) residing in orthogonal dimensions. For the photon the source is storage within time-residing radiation and the destination/release is to space-residing matter. The continuous

\footnotetext{
${ }^{4}$ There is, of course, an EM wave character as well, created by work done on a charge: orthogonal self-sustaining electric and magnetic fields that are in synch with oscillatory photon KE. These EM radiation cycles as pure occurrence reside in time but are common/present for space paths and can interact there with matter. When light enters glass the EM electric field cycles interact with the (charge bearing) electrons in the glass to slow light's progression although energy is not lost.
} 
release potential of what is stored gets mediated through the space-time separation of occurring radiation and existing matter. This accounts for noncausal, discretized release that is predictable for the aggregate but random for the instance.

\subsection{Collapse of probability waves}

We have seen that a photon's potential (relativistic) mass progressing on all available space paths is an $\mathrm{E}=$ $\mathrm{mc}^{2}$ expression of that photon's KE. This makes the entire wavefront of potential mass dependent upon a single oscillation entity in the time dimension. If that occurrence in time ceases, the dispersed potential (relativistic) mass in space disappears (ceases to occur) in its entirety. And the latter collapses instantaneously regardless of its spatial extent with no signaling required.

Instantaneous collapse happens because: 1) occurring potential (relativistic) mass carries neither energy nor rest mass; and 2) occurrence (i.e., oscillation frequency) or cessation of that occurrence in the time dimension is common to all space paths. Having something immaterial in space depend upon pure oscillation occurrence in time explains instantaneous collapse. This is another strong indicator that photon energy resides in time rendering its oscillation common to all space paths.

Local collapse: Assume some of the available space paths for photon potential (relativistic) mass waves are blocked by a detector. If the photon KE does not register (terminate) on that detector, then those blocked waves will collapse without a trace. Waves that cannot progress in space cease to occur.

General collapse: At photon termination all remaining photon potential (relativistic) mass waves will collapse regardless of how widely dispersed they are.

Collapse can only happen on a material object at a space point. When a general collapse and termination does occur, it is the photon's KE that reclaims what is common to all available space paths. The result is the delivery of the photon's undiminished KE (and momentum) to a space point on the target. Nature is clever and subtle.

\section{PHOTON AS PARTICLE?}

Regarding the photon as a real, path-traveling particle runs into various paradoxes. Nevertheless, it is still a popular concept for physicists. Classical physics has KE as a quantitative payload for something moving in space and quantum physics has never really challenged that for massless radiation. Of course there is the comparison with the electron, based on their shared wave behavior. But to lump the photon and the electron together as examples of waveparticle dualism is too simplistic. The photon has only waveform; its only presumed particle nature is termination at a point and that is misinterpreted. The electron truly has both wave and (rest mass) particle features. The electron can leave a trace in a cloud chamber because it follows a trajectory; the photon does neither.

Physicists get away with calling the photon a particle because the photon's quantized energy acts like a particle when interacting with their instruments. Abraham Pais [5] writes that although the photon has zero mass, physicists “... nevertheless call a photon a particle because, just like massive particles, it obeys the laws of conservation of energy and momentum in collisions, with an electron say (Compton effect)."

Physicists want to write equations that describe the transmission of energy or force over space; that is the basis of their craft. Waves are not suitable for that since they disperse; hence "particle" is the concept of choice to traverse space. It also conforms to the universal belief in existence, mass and space as defining reality. Physicists have their computational reasons for regarding the photon as a particle, but that doesn't make it a real particle. 


\section{HOW THE PHOTON TRAVERSES THE MZI}

A single photon entering an interferometer's first beam splitter (BS) has its space-progressing potential (relativistic) mass divided in two while it time-residing kinetic energy is unaffected. If the upper path of an interferometer contains a photon detector (obstacle), the photon's (reduced) Shoton $\rightarrow$ BS potential (relativistic) mass wave front will reach it but with only a $50 \%$ chance of terminating on it. If photon reception (termination) does not take place on this (blocking) detector, then local collapse of these blocked waves occurs (Section 4.2). This means the lower path instantaneously (non-locally) converts from $50 \%$ to $100 \%$ probability of photon reception. Stored (relativistic) mass progressing on space paths and constituting an immaterial, waveform, probability occurrence can be retracted instantly if it cannot oscillate and hence cannot progress on that path due to an (opaque) obstacle. Stored (potential) mass is a collapsible "ghost

wave" of probable release. ${ }^{5}$

A single photon allowed to traverse both arms (no blockage) of a Mach-Zehnder interferometer yields wave information (interference) when it encounters the second beam splitter. This is because the single photon's potential (relativistic) mass - divided by the first beam splitter - undergoes interference when reunited by the second beam splitter. If you place a detector on one arm of the interferometer to obtain "which-way" (which path) information, you block the passage of the photon's stored (relativistic) mass on that arm. If the photon does not register (terminate) on that detector it is a mistake to conclude that nothing was on that path and that the photon chose the other path. If the photon does register on that detector it is equally a mistake to conclude that nothing traversed the other path.

Wave interference occurs in space; it is the space-progressing potential (relativistic) mass of the photon that produces this. Blocking the passage of photon's potential (relativistic) mass wave on one arm prevents any wave interference at the second beam splitter. Similarly, for a photon traversing a double slit, positioning a detector behind one slit has the same effect as a detector on one arm of an interferometer; the wave pattern disappears. Space location ("which-way") and wave interference phenomena (multiple paths) are mutually exclusive. The blocking of probability wave paths constitutes a physical change for radiation even if photon reception does not occur on the blockage. ${ }^{6}$

It was, and remains, a great mystery that a photon seems to adjust its behavior - interference or no interference - when an experimenter places or removes her measuring (blocking) device on one path even when that device fails to receive a photon (i.e., a termination). This has occasioned many "which-way" experiments over the decades. Certainly the most famous was suggested by John Archibald Wheeler. His delayed choice thought experiment [8] has generated a huge literature and several attempts to carry it out in practice.

Wheeler theorized that the photon made a defined choice at the beam splitter: follow both paths as wave, or follow a single path as particle. Subsequent to that choice the observer might insert (activate) detectors on the two paths to measure (receive) a particle, or, retract (deactivate) those detectors and measure a wave (interference at the second beam splitter). The observer's role in determining wave versus particle aligns Wheeler with his mentor, Bohr, who argued that reality depends upon how we decide to measure it, a view anathema to Einstein. For Wheeler the role the observer plays implies retrocausality: the first beam splitter's choice gets determined by the subsequent observation

\footnotetext{
${ }^{5}$ The term "ghost wave" is from Einstein; see [6]. His instincts, as usual, were correct about a retractable wave of probability. But the irony is that Einstein tried to eliminate relativistic mass. Perhaps his biggest mistake was not the cosmological constant.

${ }^{6}$ Such a physical change is the basis of interaction-free measurement. See Elitzur-Vaidman bomb tester [7]. Note: "Tagging" photon potential mass with polarizer filters (horizontal filter on one arm, vertical filter on the other) also doesn't work; the interference pattern disappears.
} 
choice. This led Wheeler to claim that "we...have an inescapable, an irretrievable, an unavoidable influence on what we have the right to say about what we call the past."

Wheeler was wrong; there is no such thing as retrocausality. When particle detectors are placed on both paths only one detector will receive the one photon KE. This leads to the too-easy assumption that nothing travelled the other path. In fact, the photon's potential (relativistic) mass travelled both paths. Once again the mistake here is to limit the photon to but a single identity and overlook its probability identity (potential mass). The in-flight photon is a pure, waveform occurrence whose time-residing energy never divides in space, but whose objective/occurring probability wave does. The naïve idea that anything "real" will register on our material detectors on a known path discounts probability waves. These waves are physically real, occur and make their own arbitrary choice as to whether to register or not and if not then to collapse without a trace.

\section{CONCLUSION}

Successive generations of physicists have used the MZI (and the double slit) to investigate duality and the nature of radiation. It cannot be said that their efforts have advanced our understanding much. The photon still gets regarded as a unitary object, either as a quasi-particle or as a field disturbance. The photon remains an object that has contradictory attributes, discrete like a particle and continuous like a wave.

- The photon is not a simple object in space and time. It is an entity possessing two identities that together account for the incompatible photon attributes: discrete-particle versus continuous-wave.

- One identity is oscillation energy residing in time making it common to all available space paths and hence exempt from rarefaction.

- The second identity (due to $\mathrm{E}=\mathbf{m c}^{2}$ ) is potential (relativistic) mass in space that progresses and rarefies on all paths, determines probable reception and collapses nonlocally upon reception because it depends upon a single occurrence in time.

- Together these two identities explain all the usual MZI issues: 1) how the photon can split at the first mirror yet keep its energy undivided; 2) why an obstacle on one MZI path destroys wave (interference) behavior even when the photon does not terminate on the obstacle; and 3) why photon termination of diffracting coherent light is deterministic in aggregate but random for the instance.

- Radiation is the realm of quantized entities requiring time to occur (cycles); matter is the realm of quantized entities requiring space to exist. We have interpreted radiation with the concepts used for matter; this required energy to be a quantity with no presence in a dimension. This mistake leads to numerous paradoxes.

\section{CONCEPTUAL OBSTACLES}

Photon physics is not easy; that is the first obstacle. The difficult concepts in these pages include orthogonal identities, kinetic energy residing in time, nonlocal collapse, probability waves, and occurrence-energy-time as the

\footnotetext{
${ }^{7}$ Wheeler's oddly-worded statement $[9$, p.6] stops just short of asserting that we can change the past. His mentor did not excel at clarity either! For views of Wheeler, Bohr and Einstein see [10].
} 
equal of existence-mass-space. But without these concepts we have no explanation as to why the photon rarefies in space yet keeps its energy intact.

Another obstacle is our very human tendency to apply the constructs and objects we are familiar with to the realm of radiation. We are heir to a $19^{\text {th }}$ century concept of kinetic energy as a formless quantity with no dimensional presence and no oscillatory character; it does not serve us well in the case of the photon. Radiation is the transmission of kinetic energy through space and a different concept of kinetic energy is required there, one that incorporates oscillation. But it is so easy and comfortable for us to think that reality as limited to existence, mass and space with kinetic energy as quantity forced to fit in as best it can.

Retaining the traditional view of kinetic energy as quantity and continuing to apply material world concepts to radiation leaves us unable to explain something as simple as the MZI. In lieu of an explanation we resort to paradoxes or to undetectable fields with properties convenient to requirements. Old and familiar ideas are comfortable; change is difficult; that is a final, big obstacle. As Abraham Pais writes, "Like most of humanity, physicists tend to cling tenaciously to what they know or think they know, and give up traditional thinking only under extreme duress [11]." 


\section{Appendix A Topics not covered}

\section{PHOTON ENTANGLEMENT}

A principle concept here has been the ontological equivalence and equality of rest mass particle entity in space with photon KE entity in time. Each is quantized and each has a kinetic (unstored) and potential (stored) identity. Given their equality, each should bond to like instances in the same manner allowing for the inversion that existence vs. occurrence requires. If particles or atoms can bind in space via shared potential energy, then photons can bind in time via shared potential (relativistic) mass. If one pair bonds and resides in space as existing mass-based entities, then the other pair bonds and resides in time as occurring KE-based entities. When entities bond they are part of a common object, something familiar to us for material objects. When KE-based entities ("photons") bond they are part of a common event (e.g., parametric down-conversion). Mass-based entities and KE-based entities bond in but one dimension, space and time respectively. This makes them common to (shared by) observers in the alternate dimension. Space-entangled atoms/molecules are common to time-separated observers; and time-entangled photons are common to space-separated observers.

Space-adjacent, existing particles will bond via existing potential energy: atoms bond via the sharing of valence electrons; electrons possessing a negative charge bind to a nucleus with a positive charge. Binding in space accords with our existence-mass-space view of reality; binding in time is equivalent, but it is alien to us.

Assume energy is added to a calcium atom promoting two $4 \mathrm{~s}$ electrons to the (unstable) $4 \mathrm{p}$ orbital. When that energy is released the two electrons return to the $4 \mathrm{~s}$ orbital and generate two (entangled) photons, one blue and one green in frequency. The blue and green photons are created as unitary energy change in a cascade: each is a KE-asentity in time; they are time-adjacent; and they are linked (bonded) by their shared, occurring potential (relativistic) mass. Their potential identities - waveform stored mass - progress in space and overlap (superpose) as waves do. As we have seen (Section 4.2 above), these waves of probability are subject to instantaneous collapse.

The blue and green photon KE entities reside in time, are bound there and their spin orientation as a unit is undefined since spin is individual. Meanwhile their merged potential (relativistic) mass waveforms progress in space at the speed of light. Because the two photon KE entities reside in time, they are common for (available to) all points on space paths their potential identities follow (Section 3. above). At some distant point the blue photon's potential mass may trigger a blue photon reception and several things then happen. The blue photon's KE is transferred from time to a space target/detector via a space/time event (absorption). The blue photon's potential (relativistic) mass waves collapse instantly, nonlocally regardless of extent. In addition, the spin of the blue photon's KE is defined which thereby instantly, nonlocally defines the spin of its time-conjoined partner. No space signal is required to orient the green photon KE's spin since the two photon kinetic identities are not space separated. This concept is completely foreign to our classical ontology and its concept of photons as quasi-particles with a defined space location and an energy payload.

The current (existence, mass and space) assumption and mistake is to imagine that a unitary photon object travels space paths and its termination point marks its final space location. Instead, photon entity KE resides in time where it is stationary and it never travels space paths although it is common to them, just as the inertial chair you sit in is stationary in space and is common to time paths/locations. Photon KE-as-entity is never in space in the sense of occupying a volume there and having a defined location. But at termination the KE itself gets transferred to a space 
target. At the point of reception (at a space point) photon KE has ceased to be an entity; it is now an event (two entities interacting). Photon KE becomes one participant in a probabilistic, quantized, random intersection of occurrence with existence.

Photon entanglement as a natural result of $\mathrm{KE}$ entities residing in time is quite straightforward: all like entities can bond in the dimension where they reside. There is another issue that is also easy to understand. Probability waves in space (where they are subject to diffraction) must collapse nonlocally upon photon reception. They can only do so if they depend upon a single KE entity residing in time.

The fact that putting $\mathrm{KE}$ in time can resolve both these issues in a straightforward manner is something to keep in mind while navigating the more difficult issues that follow.

So receiving paired photons individually at widely separated space locations does not mean that the two photon KE entities are space separated. It is like having sodium bonded to chlorine (both particle entities) as a spaceresiding unit. Measuring each constituent at different time locations does not mean they are time separated; it means as space-residing existents they are common to all (available) time locations. Entangled photon energies are time-residing occurrences common to all (available) space locations.

- Photon entanglement is the best proof we have that $\mathrm{KE}$ is an entity residing in the time dimension and able to bond there.

- The essence of a photon (its 'kinetic' identity) resides in time and can bind there to another. The reception of one photon at one location defines spin for both photons without any signaling or coordination across space.

So congratulations Albert E. Your instincts were correct as usual; nonlocality is not what everyone (even the reluctant John Bell) thought. There is no "spooky action at a distance" and special relativity is not violated. All those critics who said you were wrong were themselves wrong. And you met that a few times in your life.

\section{ELECTRON DUALITY}

When accelerating a stationary electron two resistive forces are encountered. First there is the inertia of the electron's rest mass. Second there is a recoil force on the electron's electric field because accelerating it leads to an emission of radiation energy (Abraham-Lorentz force). Overcoming these resistive forces requires work which creates KE some of which is associated with the electron's rest mass and the rest with the EM radiation generated. Although both rest mass and electron charge have a claim on the work done (i.e., the KE), there are not two kinds of KE. And since photon KE is an entity in time, then so is the KE of the electron's rest mass. ${ }^{8}$ Matter-in-motion is not what we have always assumed, namely the adding of KE-as-quantity to rest mass. Rather, matter-in-motion is the union of two separate entities: existing, non-oscillating rest mass entity residing in space plus occurring-oscillating KE entity residing in time.

\footnotetext{
${ }^{8}$ Photon KE and the KE of matter-in-motion are indistinguishable as entities in time and both have a potential (relativistic) mass waveform in space. But their space expressions differ since KE is acting on different space-residing "objects" (rest mass vs. charge field). Photon KE-as-entity has a space component of oscillating, orthogonal, in phase electric and magnetic fields in space that enable spin, polarization and propagation at the speed of light. Rest mass electron KE-as-entity has as space component the electron's rest mass. Photon potential mass is a waveform; the electron's potential mass is a de Broglie wave packet.
} 


\section{IIa De Broglie radiation}

The wave nature of matter-in-motion began with Louis de Broglie in 1924. He was more of a speculative philosopher than a physicist. He believed that light quanta had (very tiny) rest mass which was not constant and that particles could be regarded as thermodynamic machines [12, p,1054]. He took one incorrect assumption - that waveform light had a (rest mass) particle nature - and argued the reverse, namely that rest mass particles must have a waveform nature. He equated particle intrinsic mass where time has no relevance to a radiation wave of energy $h f$ where time is all-important; this did not make much sense. ${ }^{9}$ De Broglie's 1924 thesis constitutes a “...barrage of novel ideas and confusing developments ... [12]." Textbooks and historians of physics rightly laud de Broglie for opening the way to a true quantum theory. But those few [13] who examine his thesis closely agree that his (shifting) arguments do not support his conclusion; he achieved spectacular success based on wrong supporting ideas. Physicists have never paid much attention to de Broglie's theoretical arguments; their focus has been on the detection of electron waves (Davisson and Germer) and then the Schrödinger wave equation. As a result we don't have a convincing explanation for matter waves. But the reason that both EM radiation and the moving particle (electron) have wave characteristics is much simpler than de Broglie imagined. Both the photon and the moving particle involve work done and the resulting KE is always oscillatory.

\section{IIb Mixed entities}

Pure entities were identified (Section 2.1) as: 1) the photon with KE but no rest mass; and 2) the inertial particle with rest mass but no KE. A mixed, or hybrid, entity has both rest mass and the KE of motion; the moving electron is an obvious example. Inertial rest mass and the photon are single entity objects; the moving electron is a union of two very different entities.

When moving objects are massive, then rest mass entity characteristics overwhelm the oscillatory-waveform characteristics of the conjoined KE entity. But when rest mass is vanishingly small (e.g., an electron) then waveform $\mathrm{KE}$ comes to the fore. Electrons are never quiescent so they always have significant KE and a resulting de Broglie wave character in space. High speed electrons have more KE relative to their rest mass; but their wave character becomes harder to measure. This is because cycle wavelength and particle momentum are inversely related; relatively low speed electrons with longer wavelengths make diffraction detection easier. That worked well for Davisson and Germer as their electrons were relatively slow.

\section{IIc Interaction of time-residing $\mathrm{KE}$ with space-residing rest mass}

As a mixed entity, a moving electron has its KE entity residing in time and its conjoined (rest) mass entity residing in space. It might appear they could not interact. But KE by itself has momentum due to the relativistic mass it generates via $\mathrm{E}=\mathrm{mc}^{2}$ (the photon has mass/momentum without rest mass). The electron's rest mass also has momentum since it has velocity. Momentum is both a quantity and a vector. It is a quantity dependent upon both mass and velocity; it is also a vector with a space orientation. The momentum of waveform potential (relativistic) mass has a constantly changing vector orientation; this fluctuating directionality is impressed upon (merged with) the electron's rest mass momentum. As a result the electron's rest mass will imperfectly take its momentum direction from waveform potential mass; and the smaller the rest mass the more perfect is the waveform of the rest mass. The

\footnotetext{
${ }^{9} \mathrm{E}=\mathrm{mc}^{2}$ tells us that mass and energy store each other. If energy $\mathrm{E}$ is KE (not stored), then the mass $\mathrm{m}$ is stored (relativistic mass). Conversely, it says that if mass $m$ is rest mass (unstored) then energy $E$ is stored (intrinsic energy, not KE). De Broglie equated rest mass which is unstored with radiation KE which is also unstored.
} 
Hamiltonian energy equation allows us to model the electron's potential mass waveform as a result of momentum sharing; from this we can predict electron rest mass space paths/locations in the aggregate.

Photon Double Slit: $\quad$ Work-created KE-as-entity always generates potential (relativistic) mass waves that progress in space. ${ }^{10}$ Commentators write about the "photon" entering a double slit as if the photon is unitary. Actually photon $\mathrm{KE}$ is in time (where it is stationary) and it cannot physically pass through the slits. That is the function of the photon's potential (relativistic) mass as waveform. Those waves interfere and create paths of differing intensity. That dispersed intensity determines the local probability of time-residing photon KE crossing over to space-residing matter (Section 4.1).

Electron Double Slit: For the electron its rest mass will choose one slit or the other. But it is the electron's potential (relativistic) mass as de Broglie wave that passes through both slits, undergoes interference and determines the local probability of electron rest mass reception via momentum sharing. We can write a wave equation $\Psi$ that models these electron probability values, although the mathematics requires multi-dimensional space and no one currently understands quite what the equation is modeling and why it must collapse. ${ }^{11}$

\section{IId De Broglie wave binding}

When the KE entity is on its own (photon case), we have seen that it can bond in time to a similar KE (photon) entity creating an entangled whole. The bond is created by a sharing of the potential (relativistic) mass each KE entity possesses. When a KE entity joins with rest mass (the moving electron case), the resulting oscillation of the rest mass becomes an additional source of sharable, potential (relativistic) mass. That is, both KE on its own and the oscillating electron rest mass generate relativistic mass; one by pure energy and one by rest mass in motion. This becomes important in condensed matter physics.

Valence electrons in a crystal are free to move and oscillate but their oscillations are subject to constraints imposed by the crystal lattice that surrounds them which has its own oscillations. As a result oscillating valence electrons constitute a standing wave (a Bloch wave). These oscillation energies - of electron and of lattice - generate standing (in-place) de Broglie (potential mass) waves that interact and become resonant/coherent providing that disruptive thermal oscillation energy is kept to a minimum (by cooling). Oscillation resonance lowers the energy level of adjacent electrons indicating they are bound; they are known as "Cooper pairs" after their discoverer. This binding overcomes electron charge repulsion and takes place even though the electrons are separated by many lattice intervals and even though pairing is not permanent.

When a conductor is cooled, the free electrons form a coherent waveform state with the same phase in time. With zero resistance to electrical current they constitute a superconductor whose essence is "coherence between the de Broglie waves of all the Cooper pairs [14, p. 109]." Because electrons are dominated by their de Broglie waveform character, electron wave coherence bonding is not limited to free (valence) electrons. Bound electrons in the shells of a multi-electron atom also interact with each other via oscillation resonance.

The interpretive mistake we have seen before - regarding the photon as unitary - surfaces for the electron as well. Commentators and experimenters say the "electrons are entangled over space." In fact each electron has a KE entity whose oscillation has become resonant with others. ${ }^{12}$ Two electrons without KE would never bond; they would

\footnotetext{
${ }^{10}$ The KE of a vibrating electron generates an in-place, standing wave, next section.

${ }^{11}$ It models something potential, progressing in space that depends upon a KE entity in time. This makes it subject to space path disruption (via pinholes, slits) while still having a single point of failure in the time dimension. It diffracts with its potential identity; it terminates with its kinetic identity.

${ }^{12}$ Do Cooper pairs bond via resonant oscillation or via potential mass momentum synching? Let the reader decide.
} 
simply repel each other. The electron is a mixed entity of energy and mass. The KE entity enables the binding; the electron rest mass entity is simply "along for the ride."

Rest mass entities binding in space (e.g., hydrogen binding to oxygen) is conceptually the same as KE entities binding in time. But we will forever treat the latter as "spooky" and amazing because we live and think in a world of existence, mass and space.

\section{IIe Duality, uncertainty and collapse}

Duality: We have seen (Section 5) that wave-particle duality for EM radiation depends upon a specious analogy: reception of pure, time-residing KE upon space-residing target is interpreted as 'impact.' Pure entities do not have a dual character. Thus space-residing rest mass has a particle nature and time residing photon KE has a oscillatory/wave nature. ${ }^{13}$ It is mixed entities - e.g., the progressing or orbiting or vibrating electron - that have both wave and particle characteristics. Recognizing this goes counter to much we have learned in modern physics. It is so easy (lazy) and comforting to rely on the familiar mantra: "particles have waves and waves have particles."

- The photon appears to have a dual nature because its potential (relativistic) mass constitutes a wave of probability while its time-residing $\mathrm{KE}$ can only intersect orthogonal matter at a spacetime point.

- The electron truly has a dual nature since it is a joining of existing rest mass with occurring KE.

Uncertainty: This is usually attributed to the wave nature of matter. A more fundamental reason has to do with the presence of KE. Without KE, matter has no wave nature, and hence no uncertainty. Work done on a particle creates a potential mass waveform. But this waveform is accompanied by particle rest mass which makes for multiple wavelengths (a wave packet); this renders a wave measure imprecise. Meanwhile, the presence of KE and its oscillatory waveform momentum makes a particle location measure imprecise. Eliminate the rest mass and the wave measure (wavelength) is precise (a photon); eliminate the KE and the particle's space measure (location) is precise (an inertial particle). But for a mixed entity it is always a trade off. Uncertainty is entity-based; it is ontic not epistemic.

Uncertainty results from blending existence with occurrence at the quantal level where the two are comparable in effects. It is not universal; it does not apply to pure entities.

Collapse: When work is done on rest mass, KE is created which is always oscillatory. The stored, potential (relativistic) mass of this KE progresses in space creating what we call de Broglie waves. The collapse of de Broglie waves is essentially the same as the potential mass wave collapse we saw with the photon giving up its KE (Section 4.2). The collapse of the wave function $\Psi$ mirrors the collapse of something physical and space-present, albeit something stored, probabilistic and occurring.

\section{MEASUREMENT PROBLEM AND SCHRÖDINGER'S CAT}

Schrödinger assumed that the electron actually was a wave that his equation described; others took the electron to be space-discrete and had to connect it and the wave equation probabilistically. In reality his equation

\footnotetext{
${ }^{13}$ A wave is a form characterizing radiation or water; a particle is an entity, not a form. A particle's form would be "fieldform" or "matterform" but neither will catch on. We are stuck with "particle" being both entity and the form said entity possesses.
} 
describes the space-progressing, waveform, probabilistic stored mass of the KE-as-entity; the latter having joined with the electron's rest mass via work done. To repeat: the Schrödinger wave equation is modeling KE's relativistic mass which has the waveform and not the electron's rest mass which has the particle-form. The idea that the wave function must describe the electron's rest mass follows from the (classical) assumption that both KE and the (relativistic) mass it stores are formless quantities with no presence in a dimension. Attributing wave character to the rest mass particle made the latter appear as probabilistic in its existence or its space location. From this disconnect endless difficulties of interpretation have arisen over the years. Known collectively as "the measurement problem," these difficulties have led to numerous questions. Why is collapse necessary and what does it mean? Why only probability values from our equation? If rest mass (the electron) can be smeared over space how do we get from there to matter being discrete? How can we get real knowledge of the quantum world using instruments obeying classical physics?

The 'solutions' of the measurement problem over the decades extrapolate from our common experiences rather than looking at ontology. Working physicists ignore the problem entirely ("shut up and calculate").

Photon and electron wave collapse have already been explained (Sections 4.2, IIe) as a consequence of occurring (waveform) potential mass in space being dependent upon an occurring entity in time. Remaining issues can be addressed by looking at the theory of particle superposition and the famous thought experiment it spawned.

Explaining how the discrete electron can act like a continuous wave has led to the theory of superposition. Waveform superposition is something well-known and universally accepted both physically and mathematically. Waves can overlap in space and reinforce (or negate) each other. But applying superposition to material entities means an existing rest mass can be in two places or states at once. This 'solution' got a bit of mild mockery from Erwin Schrödinger when he published his famous thought experiment involving a cat that was both dead and alive. Schrödinger's thought experiment projects the probabilistic state of an unstable atom on to a cat and the details are familiar to anyone who has gotten this far.

Assume there is a heavy atom (many electrons/protons) with an alpha particle (two protons and two neutrons bound together by the strong force) oscillating within the potential well of the nucleus. Like the much lighter electron, the energetic alpha particle has KE as an entity joined to its rest mass. The KE-as-entity has its stored (potential) mass joined to the alpha particle rest mass entity as a standing wave which can be modeled by the wave function $\Psi$ [15].

The wave function for the confined alpha particle yields a smeared probability density field for the particle's position. A portion of this field will extend beyond the potential barrier limits (quantum tunneling). From this a probable particle release (decay) rate per hour or per day may be calculated. If the alpha particle remains in the nucleus the cat lives; if the alpha particle escapes the nucleus the lethal causal chain (detector, hammer, poison) is triggered and the cat dies. The wave function shows both cases (solutions) simultaneously and so the inference is that the cat is both dead and alive; these states are superposed.

By now the astute reader can see the way out of this paradox. The cat's state depends on the location of the alpha particle's rest mass: either inside the nucleus (alive) or outside the nucleus (dead). But the wave function is only characterizing the stored (relativistic) mass of the KE-as-entity, the latter having joined with the alpha particle's rest mass via work done. While a portion of the standing wave of KE's potential mass may be outside the potential barrier of the nucleus, said potential mass waveform is merely objective probability (Section 4.1). This potential mass waveform has no connection to, or effect upon, the causal chain that kills the cat; it is the rest mass that has that connection. Put another way, the wave function does not apply to (does not model) that entity (the alpha particle's rest mass) that can lethally affect the cat. Arguing that superposition allows a rest mass particle to have two space locations at the same time reeks of explanatory desperation. Superposition applies to waveforms, in this case to space-continuous potential mass as wave. Superposition does not apply to material, particle-form entities such as a cat or an alpha particle rest mass. The cat is never in a mixed, dead/alive, state. Curiosity kills cats, not probability waves. 


\section{REALISM IN PHYSICS}

Niels Bohr saw a clear distinction between the quantal world of electrons and light quanta with the classical world of our instruments and measurements. He didn't think either one could tell us something about the other; he would not interpret quantum randomness to imply universal randomness. How ironic then that his own student, J. A. Wheeler [9, p.9] would come to write "There is not a single sight, not a single sound, not a single sense impression which does not derive in the last analysis from one or more elementary quantum phenomena." Steven Weinberg [16] agrees: "Physicists and their apparatus must be governed by the same quantum mechanical rules that govern everything else in the universe. But these rules are expressed in terms of a wavefunction...."

This is ontological reductionism: the argument that entities at a certain level can only be understood as collections or combinations of simpler entities at even lower levels. The "ultimate laws of nature" therefore operate at the very bottom of the hierarchy of being.

Ontological reductionism has its place in scientific inquiry but it needs to be balanced by recognition that properties can emerge from the whole and not from the parts. Temperature characterizes a gas but not individual gas molecules. Saltiness characterizes sodium chloride, but not sodium nor chlorine. 'Orbiting' electrons and their vibrating atoms/molecules are as much (or more) occurrence than existence. But this activity is energy that actually binds constituents together so several levels up they constitute stable, existing matter familiar to us. What is discrete, oscillating, and kinetic energy at the lowest level becomes continuous, existing and potential energy only a few levels up. Those who deny emergent properties and insist that the wave function applies to human scale material objects are making an expression of faith that is based on an uncritical acceptance of reductionism.

Niels Bohr also counseled a generation (or two) of physicists to refrain from speculating about the quantal world. But the human mind is not built that way. The impossibility of explaining quantum mysteries and paradoxes within the existence, mass and space ontology has led to much speculation; especially since the 1970s. Some of this speculation may have some merit although it is hard to find. It has been called "fairy tale physics." 14 All ages have their silly ideas; ours may be unique in the brain power (or at least the training) of the advocates. As an antidote, let's see if we can interpret reality based simply on what we know: mass, energy, space, time and the concept of entity; with mass and energy treated as primitives.

\section{IVa Different physics for different entities}

Section IIb points out that ultimately physics studies entities of three types. Not surprisingly, each type has its own form (particle-form, waveform, mixed) and, in broad outlines, its own physics. Pure rest mass entities (inertial matter, no KE) have the particle-form and obey classical physics. Pure KE entities (EM radiation quanta) have the waveform and obey classical optics and electrodynamics (Maxwell's equations). Mixed-form entities obey wave mechanics providing the radiation (KE) entity is significant relative to the rest mass entity (i.e., typically if the rest mass is tiny). There are a few necessary qualifications.

Classical physics is perfectly valid for stationary material objects above the quantal level. ${ }^{15}$ Thus material objects, regarded as media, obey familiar/classical equations for statics (distribution of forces), stress/strain (Hooke's law) and hosted waves (sound waves, water waves, etc.). If the medium itself is uniform then the equations are straightforward; otherwise they are the sum of local calculations. Classical mechanics (moving bodies) is very good (not perfect) for matter-in-motion providing velocity is low and rest mass is large.

\footnotetext{
${ }^{14}$ E.g., multiverse, supersymmetry, braneworlds, etc. See [17, p.xii, 180-202, 284-7]

${ }^{15}$ At the quantal level no objects are stationary; energy dominates.
} 
Classical optics and electrodynamics are perfectly valid for radiation except when photons interact with subatomic particles since this evokes mixed-entity behavior requiring quantum mechanics. Such interactions involve charges (and their tiny masses) interacting at small distances and low field strengths making QED necessary. This same limitation applies to condensed matter physics.

In general classical physics applies to pure form entities. It is wave mechanics that absolutely depends upon a lack of purity of form and that is beyond dispute: wave mechanics cannot be used for pure form entities, namely inflight EM radiation or a boulder with zero momentum. Wave mechanics and Heisenberg uncertainty require the joining of matter (rest mass) and radiation (KE); remove the rest mass entity or remove the KE entity and wave mechanics and uncertainty no longer apply.

Of course, quantum physics is broader than wave mechanics and includes quantum field theory (QFT). This theory extends Schrödinger's wave mechanics to make it: 1) relativistic; 2) capable of dealing with many particles; and 3 ) cover the creation and annihilation of particles. But classical physics is for entities that exist and entities that occur and by definition does not cover (1) their blending; or (2) their transitioning (creation, annihilation).

The question as to what separates classical physics from quantum physics has been a source of controversy for about a century. As noted, the temptation to regard wave mechanics as foundational (the "queen of physics") has proved irresistible for most. Despite Wheeler [9, p.9] and Weinberg [16] as quoted above, our laboratory instruments are not governed by quantum mechanical rules nor are they subject to the Heisenberg uncertainty relation. The electrons in our instruments may be in constant motion and obey the wave function but several levels up this electron KE has become binding energy in a static crystalline structure which exists.

Quantum physics is very different from classical physics and for a good reason: they describe different entity configurations. There is a defined, ontological separation between the microworld and the macroworld; it is based not on size but on pure entities versus mixed entities. Viewing KE as a quantity and not an entity has prevented us from seeing that.

- Quantum mechanics does not tell us what ultimate reality is like; as a science it is valid for that very limited transition region where mass is losing its soul to energy. Physical reality (which involves presence in a dimension, matter in space and energy in time) is much broader than this transition region.

- It is a serious mistake to search for, and enunciate, fundamental truths about physics and reality on the basis of mixed entities plus reductionism.

Reflections: $\quad$ Transforming KE from a quantity to an entity in time affects many areas of physics. On the plus side it resolves most of the mysteries and paradoxes that have surrounded quantum mechanics for over a century. It provides the first real explanation of photon entanglement and shows that nonlocal, instantaneous change is not what we thought it was. Hopefully this is evident (or plausible) for the reader despite the author's many obscurities or mistakes. On the minus side a lot of new concepts are involved and no new predictions emerge that might be tested in the laboratory in the manner that Bell's theorem was tested. For these reasons those who study and work with physics (physicists, philosophers) will not be disposed to abandon their current view of KE as a quantity since, as noted, people “...give up traditional thinking only under extreme duress [11]." For most people (not all), doing a lot of (mental) work to solve a paradox or two while upsetting a familiar concept of energy first learned as a teenager is a bad bargain. Especially true if you hold or have published quantum theories that depend upon the existence, mass and space ontology. 
Particle physics aligns with how we view reality (our surroundings) and so it will continue to dominate for that reason plus academic/institutional inertia and funding. Nobel prizes will continue to be awarded for new 'particles' added to a standard model, a model that cannot predict/calculate the masses of its identified particles. The current count is 61 'elementary' particles and some impressive results have been teased out them. But this very complicated standard model of particles is built on the existence, mass and space ontology. So of course it cannot tell us what the wave function represents, how/why collapse occurs or how the photon navigates the MZI. 


\section{REFERENCES}

[1] Danan, A, Farfurnik,D., Bar-Ad, S. and Vaidman, L. "Asking photons where have they been," Phys. Rev. Lett. 111, 240402, (9 December 2013). Also: arXiv:quant-ph/1304.7469 v2.

[2] Peleg, U., Vaidman, L., "Comment on 'Past of a quantum particle revisited'," Physical Review A 99, 026103 (2019), also https://www.tau.ac.il/ vaidman/lvhp/m156.pdf (May 2020).

[3] Koks, Don, "What is Relativistic Mass?” http://math.ucr.edu/home/baez/physics/Relativity/SR/mass.html https://www.tau.ac.il/ vaidman/lvhp/m156.pdf (10 December 2019).

[4] Shimony, A., "The Reality of the Quantum World," Scientific American, v258 n1, 48 (Jan. 1988).

[5] Pais , A., [Niels Bohr's Times], Oxford Univ. Press, 350-1 (1991).

[6] Dongen, J. van, "The interpretation of the Einstein-Rupp experiments and their influence on the history of quantum mechanics,” arXiv:physics/0709.3226, 2-3, (3 January, 2020).

[7] "Elitzur-Vaidman bomb tester," https://en.wikipedia.org/wiki/Elitzur\%E2\%80\%93Vaidman_bomb_tester. (4 February 2020).

[8] Wheeler, J., A., "The 'past' and the 'delayed-choice' double-slit experiment," Mathematical Foundations of Quantum Theory, ed. A.R. Marlow, Academic Press, New York, 9-48 (1978). For a summary see https://en.wikipedia.org/wiki/Wheeler\%27s_delayed_choice_experiment\#Cosmic interferometer (4 May 2020).

[9] Wheeler, J., A., "Hermann Weyl and the Unity of Knowledge, http://www.weylmann.com/wheeler.pdf (18, February 2020).

[10] Roussel, P., Stefan, J. "Is the Interpretation of Delayed-Choice Experiments Misleading?" arXiv:physics/0706.2596. (6 January 2020).

[11] Pais, A., [Inward Bound], Oxford Univ. Press, New York, 137 (1986).

[12] MacKinnon, E., 1054. "De Broglie's thesis: A critical retrospective," American Journal of Physics, 44, 1047 (1976).

[13] MacKinnon; also Roberto de Andrade Martins, "Louis de Broglie's Struggle with the Wave-Particle Dualism, 1923-1925," http://quantum-history.mpiwg-berlin.mpg.de/eLibrary/hq1 talks/waveMech/23 martins (14 May 2020).

[14] Ford, P.J., Saunders, G.A., [The Rise of the Superconductors], CRC Press, (2004). Online at: http://qudev.ethz.ch/content/courses/phys4/studentspresentations/supercond/Ford_The rise_of_SC 6 7.pdf

[15] See http://hyperphysics.phy-astr.gsu.edu/hbase/quantum/barr.html\#c1 (5 June 2020)

[16] Weinberg, S., “Einstein's Mistakes," Physics Today, v58, Issue 11, section 'Contra Quantum Mechanics,' http://physicstoday.scitation.org/doi/full/10.1063/1.2155755 (3 April 2020)

[17] Baggott, J., [Farewell to Reality: How Modern Physics has Betrayed the Search for Scientific Truth], Pegasus Books, New York, (2013) 